\title{
Dermatosis autoinmunes en caninos. Estudio retrospectivo
}

\author{
Sieben, C. ${ }^{1,2}$; Massone, A.R. ${ }^{3}$; Machuca, M.A. ${ }^{3}$ \\ ${ }^{1}$ Hospital Escuela, Departamento Clínicas, Facultad Ciencias Veterinarias (FCV), Universidad Nac. La Plata \\ (UNLP), Buenos Aires, Argentina. ${ }^{2}$ Becario UNLP. ${ }^{3}$ Laborat. Patología Dr. B. Epstein (FCV-UNLP). \\ E-mail: csieben@fcv.unlp.edu.ar
}

\begin{abstract}
Resumen
Sieben, C.; Massone, A.R.; Machuca, M.A.: Dermatosis autoinmunes en caninos. Estudio retrospectivo. Rev. Vet. 30: 1, 70-75, 2019. Se investigaron muestras de piel de caninos con diagnóstico de enfermedad autoinmune ingresadas entre los años 2004 y 2016 al Laboratorio de Patología Especial Veterinaria (FCV, La Plata, Argentina). Los objetivos fueron identificar casos de caninos con lesiones cutáneas y seleccionar aquéllos con diagnóstico de enfermedad autoinmune. También se propuso relacionar las dermatosis autoinmunes con la raza, edad, sexo, el tipo y la ubicación anatómica de las lesiones clínicas y, por ultimo, describir las diferentes lesiones histopatológicas que caracterizaron cada afección. Las enfermedades autoinmunes representaron el $2,07 \%$ del total de casos ingresados en el período de estudio, siendo las más frecuentes el pénfigo foliáceo y el lupus eritematoso discoide. Los caninos de raza pura fueron más afectados que los mestizos, siendo la región dorsal de la nariz la ubicación anatómica de mayor presentación (35,3\%). Entre los hallazgos histopatológicos más reiterados se observaron pústulas $(54,1 \%)$, áreas de separación dermoepidérmica $(45,9 \%)$ y espongiosis $(44,7 \%)$. Si bien el porcentaje de caninos con dermatosis autoinmunes es bajo, es importante incluirlas dentro de los diagnósticos diferenciales de las enfermedades que cursan con pústulas, pápulas, vesículas e infiltrado inflamatorio en la unión dermoepidérmica. La histopatología es una herramienta útil y relativamente accesible en nuestro medio, que permite arribar al diagnóstico de tales enfermedades.
\end{abstract}

Palabras clave: canino, dermatosis autoinmunes, histopatología, estudio retrospectivo.

\begin{abstract}
Sieben, C.; Massone, A.R.; Machuca, M.A.: Autoimmune skin disease in dogs. Retrospective study. Rev. Vet. 30: 1, 70-75, 2019. A retrospective study of canine skin samples diagnosed with autoimmune disease, admitted by the Veterinary Special Pathology Laboratory, was conducted between 2004 and 2016. Purposes of the study were to identify canine cases of skin lesions and to select those with a diagnosis of autoimmune disease. Autoimmune skin diseases were related to race, age, sex, type and anatomical location of the clinical lesions and, finally, different histopathological lesions characterizing each disease. Autoimmune diseases accounted for $2.07 \%$ of the total number of cases admitted in the study period, the most frequent being pemphigus foliaceus and discoid lupus erythematosus. The purebred dogs were more affected than the mixed animals, being the anatomical location of greater presentation the dorsal region of the nose (35.3\%). Among the most frequent histopathological findings were pustules (54.1\%), areas of dermo-epidermal separation (45.9\%) and spongiosis $(44.7 \%)$. Although the percentage of canines with autoimmune dermatosis is low, it is important to include differential diagnoses of the diseases that occur with pustules, papules, vesicles and inflammatory infiltrate in the dermoepidermal junction. Histopathology is a useful and accessible tool in that allows to diagnose these diseases.
\end{abstract}

Key words: canine, autoimmune dermatosis, histopathology, retrospective study.

\section{INTRODUCCIÓN}

Las diferentes enfermedades que afectan a la piel de los caninos pueden clasificarse de acuerdo a su mecanismo patogénico en congénitas y hereditarias, desórdenes de la diferenciación epidérmica, anomalías de

Recibido: 27 febrero 2018 / Aceptado: 4 octubre 2018 la pigmentación, daños fisicoquímicos, deficiencias nutricionales, enfermedades endocrinas, dermatosis inmunomediadas, enfermedades infecciosas (virus, bacterias, hongos, protozoarios, ectoparásitos artrópodos y helmintos), neoplásicas y misceláneas.

Dentro de esta clasificación, las dermatosis inmunomediadas son aquéllas que incluyen mecanismos inmunológicos en la génesis de las lesiones. Se pue- 
den incluir a las dermatosis por hipersensibilidad, las dermatosis autoinmunes y otras condiciones inmunológicas de etiología variada que incluyen sensibilidad a drogas, vacunas y antígenos diversos ${ }^{4,6}$.

En las dermatosis autoinmunes, los mecanismos patogénicos de lesión se establecen por la existencia de anticuerpos o linfocitos activados contra constituyentes propios de la piel ${ }^{7}$. Las dermatosis autoinmunes en caninos comprenden las enfermedades caracterizadas por pústulas, vesículas o bullas como lesión primaria que incluye al complejo pénfigo (pénfigo foliáceo, pénfigo vulgar, pénfigo eritematoso, pénfigo paraneoplásico), penfigoide de las membranas mucosas, penfigoide bulloso, epidermólisis bullosa adquirida y enfermedad lineal por inmunoglobulina A.

Asimismo, también se comprenden los diferentes tipos de lupus (lupus sistémico, lupus eritematoso discoide, lupus eritematoso discoide diseminado, lupus eritematoso cutáneo exfoliativo, lupus eritematoso cutáneo vesicular y lupus paniculitis) que se caracterizan por degeneración vacuolar del estrato basal e infiltración inflamatoria en la unión dermoepidérmica ${ }^{6,7}$.

Los objetivos del presente trabajo fueron: 1) identificar los casos de caninos con lesión cutánea ingresados al laboratorio; 2) seleccionar aquéllos con diagnóstico de enfermedad autoinmune; 3) relacionar las dermatosis autoinmunes con la raza, edad, sexo, el tipo y la ubicación anatómica de las lesiones clínicas descriptas; y 4) describir diferentes lesiones histopatológicas que caracterizaron cada enfermedad.

\section{MATERIAL Y MÉTODOS}

Se evaluaron los registros del Laboratorio de Patología Especial Veterinaria Dr. B Epstein de la Facultad de Ciencias Veterinarias, Universidad Nacional de La Plata, y se seleccionaron las muestras de piel provenientes de caninos con historia clínica de enfermedad cutánea entre los años 2004 y 2016. Luego se realizó la revisión de los cortes histológicos de la piel con diagnóstico de enfermedad autoinmune.
A partir de las historias clínicas se recopilaron los siguientes datos de los pacientes: raza, edad, sexo, signos clínicos, lesiones cutáneas y sus ubicaciones anatómicas. Del análisis de las historias clínicas se registraron en planillas de cálculo las lesiones primarias y secundarias, las cuales fueron relacionadas con los datos del paciente.

Se realizó la re-observación de los cortes histológicos con el fin de caracterizar y registrar las lesiones microscópicas de la epidermis, la unión dermoepidérmica y la dermis. Por último, los datos obtenidos del análisis de las historias clínicas y los hallazgos histopatológicos fueron analizados mediante un estudio estadístico descriptivo.

\section{RESULTADOS}

Enfermedades de la piel. Durante el periodo propuesto, ingresaron al laboratorio un total de 4.171 muestras de piel provenientes de caninos con historia clínica de enfermedad cutánea. Dentro del total de muestras ingresadas, el mayor porcentaje fue para las neoplasias con el 74,22\% $(n=3096)$. Para las enfermedades autoinmunes de la piel, la proporción fue del $2,07 \%(\mathrm{n}=85) \mathrm{de}$ los casos estudiados (Figura 1).

Enfermedades autoinmunes. En la Tabla 1 se presentan las diferentes enfermedades autoinmunes diagnosticadas mediante el estudio histopatológico. Los casos en que solo se llegó a un diagnóstico de pénfigo $(n=12)$ se debieron a que los datos clínicos aportados no fueron los suficientes (descripción de las lesiones, ubicación anatómica de las mismas) y, en otras situaciones, las lesiones microscópicas no se hallaron en un número considerable para poder clasificarlas.

A partir del análisis de las historias clínicas se evaluaron los datos de la reseña observándose que los perros afectados con dermatosis autoinmunes $(n=85)$ tenían entre 4 meses y 13 años de edad, con una media de 5,14 años (el 50\% de ellos entre 2,5 y 7 años (Figura 2). En el $29,4 \%$ de los casos (25/85) los pacientes eran mestizos y en el $70,6 \%(60 / 85)$ eran de raza pura.

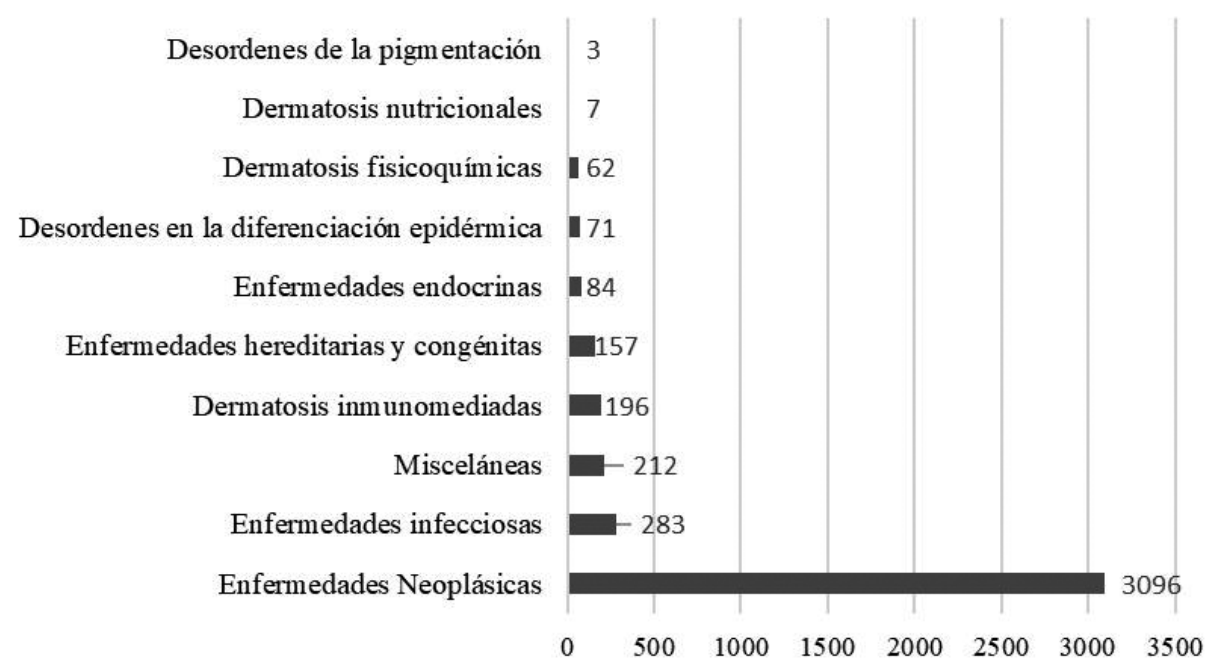

Figura 1. Total de diagnósticos de enfermedades cutáneas en caninos realizadas en el período 2004-2016. 
Tabla 1. Dermatosis autoinmunes diagnosticadas (2004-2016).

\begin{tabular}{lc}
\hline tipos de dermatosis & \\
\hline enfermedad autoinmune inespecífica & 17 \\
pénfigo & 12 \\
pénfigo foliáceo & 23 \\
pénfigo eritematoso & 5 \\
pénfigo paraneoplásico & 1 \\
pénfigo vulgar & 2 \\
penfigoide bulloso & 5 \\
lupus eritematoso discoide & 18 \\
lupus eritematoso exfoliativo & 1 \\
\hline total & 85 \\
\hline
\end{tabular}

Hubo predisposición racial para el collie (10/60), pastor alemán (10/60), cocker spaniel (6/60) y labrador retriever (6/60). En 80 perros con dermatosis autoinmunes fue registrado el sexo, resultando similar la frecuencia de presentación entre machos $(43 / 80)$ y hembras (37/80). El resultado estadístico fue no significativo (diferencia de proporciones $\mathrm{p}=0,47$ ).

Se consignaron los datos del aspecto macroscópico de la piel suministrados por el clínico y los obtenidos de los registros del laboratorio al momento del ingreso de las muestras. Entre las lesiones cutáneas primarias y secundarias halladas, las más frecuentes fueron costras $(43,5 \%)$, úlceras $(31,8 \%)$ y alopecia $(27 \%)$, mientras que las pústulas y vesículas representaron el 4,70\% y 3,53\% respectivamente.

También fue analizada la ubicación de las lesiones en la piel, las cuales tuvieron una frecuencia de presentación variable, con una tendencia a afectar la región dorsal de la nariz, denominada comúnmente plano nasal $(35,3 \%)$, la zona periocular $(29,4 \%)$ y la región de la nariz $(21,2 \%)$. Las lesiones ubicadas en las uniones mucocutáneas y en áreas de mucosa se observaron en el $27 \%$ y $16,5 \%$ respectivamente (Tabla 2 ).

En los caninos con pénfigo foliáceo $(\mathrm{PF})$ las áreas más afectadas fueron la zona periorbital, la región dorsal de la nariz y los pabellones auriculares; en los casos de lupus eritematoso discoide (LED) las zonas más afectadas fueron la región dorsal de la nariz, la nariz y la mucosa nasal (Figura 3).

En el estudio histopatológico se observó que las dos enfermedades autoinmunes más diagnosticadas (Tabla 1) fueron PF (23/85) y LED (18/85). Los hallazgos microscópicos en epidermis, dermis y unión dermoepi-

Tabla 2. Frecuencia de presentación del tipo y la ubicación de las lesiones macroscópicas en las diferentes dermatosis autoinmunes.

\begin{tabular}{|c|c|c|c|c|c|c|c|c|c|c|}
\hline tipo & EA & $\mathrm{PO}$ & $\mathrm{PF}$ & $\mathrm{PE}$ & $\mathrm{PV}$ & $\mathrm{PN}$ & $\mathrm{PB}$ & LE & $\mathrm{LC}$ & $\mathrm{TO}$ \\
\hline costras & 7 & 5 & 11 & 3 & 1 & 1 & 1 & 8 & - & 37 \\
\hline úlceras & 10 & 3 & 6 & 1 & 2 & - & - & 5 & - & 27 \\
\hline alopecía & 4 & 3 & 11 & 1 & - & 1 & - & 3 & - & 23 \\
\hline pústulas & 2 & 1 & 1 & - & - & - & - & - & - & 4 \\
\hline vesículas & 1 & 2 & - & - & - & - & - & - & - & 3 \\
\hline pápulas & - & - & - & 1 & - & - & - & - & - & 1 \\
\hline erosiones & - & - & 1 & - & 1 & - & - & 3 & - & 5 \\
\hline eritema & - & - & 5 & 1 & - & - & 1 & 3 & - & 10 \\
\hline hiperpigmentación & 4 & 2 & 2 & - & - & - & - & - & - & 8 \\
\hline hiperqueratosis & 4 & 2 & 2 & - & - & - & - & 1 & - & 9 \\
\hline descamación & 1 & - & 3 & 1 & - & - & - & 1 & - & 6 \\
\hline ubicación & EA & $\mathrm{PO}$ & $\mathrm{PF}$ & $\mathrm{PE}$ & $\mathrm{PV}$ & $\mathrm{PN}$ & $\mathrm{PB}$ & LE & $\mathrm{LC}$ & $\mathrm{TO}$ \\
\hline región dorsal nariz & - & 2 & 14 & 5 & - & - & - & 9 & - & 30 \\
\hline región de la nariz & 6 & 1 & 4 & - & - & - & - & 7 & - & 18 \\
\hline región periorbital & 3 & 3 & 13 & 1 & - & 1 & - & 5 & - & 26 \\
\hline pabellón auricular & 2 & - & 8 & 1 & - & - & - & - & - & 11 \\
\hline región de la cabeza & 2 & 2 & - & - & 1 & - & 1 & - & - & 6 \\
\hline almohadillas & - & - & 1 & - & - & - & - & - & - & 1 \\
\hline reg. tronco/cuello & 1 & 5 & 2 & - & 1 & - & 1 & 1 & - & 11 \\
\hline región axilar & 2 & 4 & 1 & - & - & - & 1 & - & - & 8 \\
\hline Reg. de miembros & 3 & 3 & 4 & - & - & 1 & 1 & 1 & 1 & 14 \\
\hline región del vientre & 7 & - & 3 & - & - & - & - & - & - & 10 \\
\hline uniones "mc" & 4 & 3 & 6 & - & 2 & 1 & 1 & 6 & - & 23 \\
\hline "mucosas" & 5 & 5 & 2 & 1 & - & - & - & 1 & - & 14 \\
\hline región inguinal & 1 & 5 & - & - & - & - & 1 & - & - & 7 \\
\hline región perineal & - & 2 & - & - & - & - & - & - & - & 2 \\
\hline
\end{tabular}

EA: enfermedad autoinmune. PO: pénfigo. PF: pénfigo foliáceo. PE: pénfigo eritematoso. PV: pénfigo vulgar. PN: pénfigo paraneoplásico. PB: pénfigo bulloso. LE: lupus eritematoso discoide. LC: lupus eritematoso cutáneo exfoliativo. TO: total. "mc”: uniones mucocutáneas (incluyen uniones labial, anal, vulvar y nasal). Las "mucosas" incluídas son: anal, oral, nasal, vulvar y prepucial. 
Tabla 3. Frecuencia de presentación de las lesiones microscópicas en las diferentes dermatosis autoinmunes.

\begin{tabular}{|c|c|c|c|c|c|c|c|c|c|c|c|}
\hline & tipo & EA & $\mathrm{PO}$ & $\mathrm{PF}$ & $\mathrm{PE}$ & PV & $\mathrm{PN}$ & PB & LE & $\mathrm{LC}$ & $\mathrm{TO}$ \\
\hline \multirow{17}{*}{ 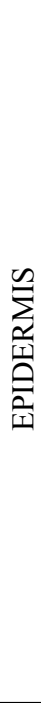 } & costras & 3 & 1 & 6 & 2 & 1 & 1 & - & 3 & - & 17 \\
\hline & hiperqueratosis & 5 & 3 & 8 & 2 & 2 & - & 2 & 1 & 1 & 24 \\
\hline & pústulas & 5 & 3 & 20 & 4 & 2 & 1 & - & - & - & 35 \\
\hline & pústulas foliculares & - & - & 10 & 1 & - & - & - & - & - & 11 \\
\hline & queratinocitos acantolíticos & 1 & 2 & 12 & - & - & - & - & - & - & 15 \\
\hline & ulceras & 6 & - & 3 & 1 & 1 & - & - & 2 & - & 13 \\
\hline & acantosis & 8 & 6 & 9 & 2 & 2 & - & 1 & 6 & 1 & 35 \\
\hline & espongiosis & 8 & 7 & 13 & 2 & 2 & - & 1 & 5 & - & 38 \\
\hline & acantólisis & 1 & - & 4 & - & - & 1 & - & - & - & 6 \\
\hline & vesículas & 3 & 4 & - & - & - & - & - & - & - & 7 \\
\hline & bullas & - & - & - & - & - & - & 4 & - & - & 4 \\
\hline & atrofia epidérmica & 4 & 1 & 1 & - & - & - & - & 3 & - & 9 \\
\hline & degen.vacuolar EG y/o EB & 1 & 1 & - & 3 & - & 1 & 2 & 9 & 1 & 18 \\
\hline & sateliosis linfocítica & - & - & - & 1 & - & - & - & 4 & - & 5 \\
\hline & separación suprabasal & - & 1 & - & - & 2 & 1 & - & - & - & 4 \\
\hline & exocitosis neutrofílica & 2 & 1 & 2 & - & 1 & 1 & - & 2 & - & 9 \\
\hline & queratinocitos apoptóticos & - & - & 2 & 1 & 1 & 1 & - & 10 & 1 & 16 \\
\hline \multirow{3}{*}{ 亗 } & separación en unión DE & 5 & 9 & 7 & 5 & - & - & 4 & 9 & - & 39 \\
\hline & infiltrado liquenoide en ZI & 3 & 1 & - & - & - & - & 1 & 11 & - & 16 \\
\hline & pérdida demarcación $\mathrm{DE}$ & - & - & - & 1 & - & - & - & 8 & - & 9 \\
\hline \multirow{9}{*}{ 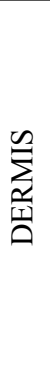 } & infiltrado en dermis leve & 2 & 5 & 4 & 1 & 1 & 1 & 4 & - & - & 18 \\
\hline & infiltrado dermis moderado & 3 & 4 & 12 & 1 & 1 & - & - & 2 & 1 & 24 \\
\hline & infiltrado dermis abundante & 9 & - & 4 & 3 & - & - & - & 4 & - & 20 \\
\hline & infiltrado PL / PA / PS & - & - & 3 & 1 & 1 & 1 & 2 & 6 & - & 14 \\
\hline & edema en dermis & 1 & 2 & 10 & 2 & - & - & - & 1 & - & 16 \\
\hline & dilat.folic.gland. sudoríparas & 1 & 1 & 3 & - & 1 & - & - & - & - & 6 \\
\hline & dilatación vasos linfát./sang. & 1 & - & - & 1 & - & - & - & 2 & - & 4 \\
\hline & hemorragia & 2 & - & - & - & - & - & - & - & - & 2 \\
\hline & incontinencia pigmentaria & 6 & 1 & 7 & 2 & 1 & - & 1 & 12 & - & 30 \\
\hline
\end{tabular}

D-E: dermo-epidérmica. EA: enfermedad autoinmune. PO: pénfigo. PF: pénfigo foliáceo. PE: pénfigo eritematoso. PV: pénfigo vulgar. PN: pénfigo paraneoplásico. PB: pénfigo bulloso. LE: lupus eritematoso discoide. LC: lupus eritematoso cutáneo exfoliativo. TO: total. EG: estrato granuloso. EB: estrato basal. ZI: zona de interfase. PL: perifolicular. PA: perianexal. PS: perivascular.

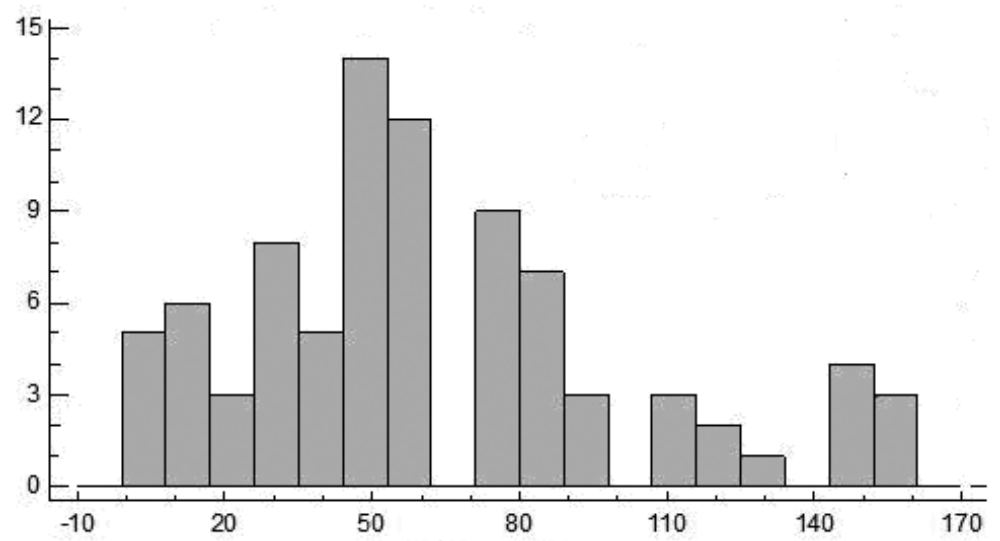

Figura 2. Frecuencia de presentación de las dermatosis autoinmunes según edad ( $\mathrm{n}=85$, media=61,69; mediana=60; desvío estándar=39,25; mínimo=4 (4 meses); máximo=156 (13 años); suma=5244; percentiles $=25-30(2,5$ años), 50-60 (5 años), 75-84 (7 años). Numeración vertical: casos registrados. Numeración horizontal: edad (meses).

dérmica se describen en la Tabla 3. En la epidermis, los hallazgos más frecuentes fueron: pústulas $(54,1 \%$, de ubicaciones subcorneales, intragranulares, suprabasales y foliculares), espongiosis $(44,7 \%)$, acantosis $(41,2 \%)$ e hiperqueratosis $(28,2 \%)$.
A nivel de la unión dermoepidérmica el hallazgo más constante fue la separación entre epidermis y dermis $(45,9 \%)$. En la dermis el infiltrado inflamatorio predominante fue mononuclear (células plasmáticas, macrófagos y linfocitos) aunque -en ocasiones- se pre- 


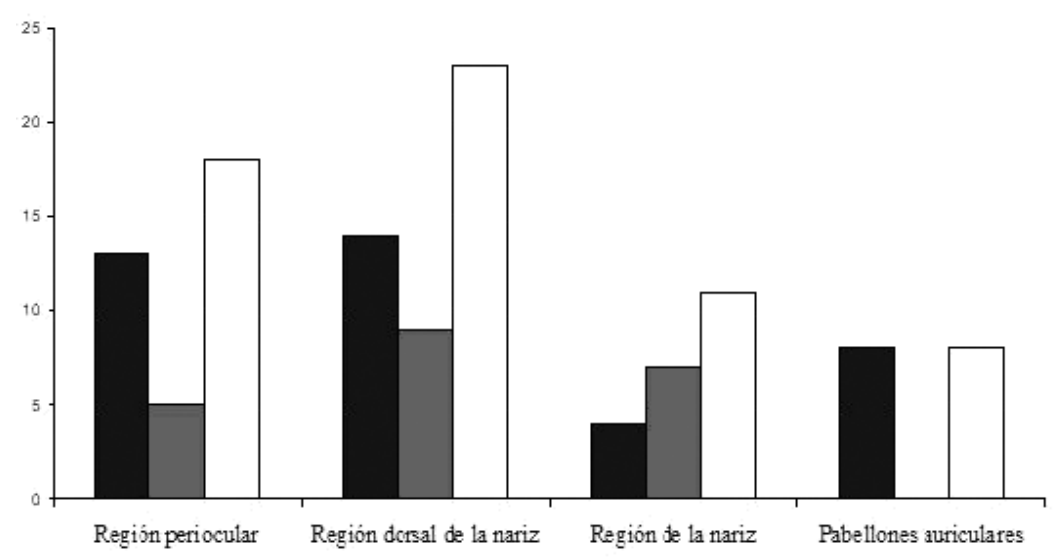

Figura 3. Ubicaciones anatómicas de las lesiones más frecuentes en PF Y LED. Números indican casos registrados. Barras negras: pénfigo foliáceo; grises: lupus eritematoso discoide; blancas: total.

sentó un infiltrado inflamatorio de tipo mixto (con presencia de neutrófilos).

Dado que se observó una gran variación en la cantidad de células que infiltraban la dermis, se realizó una clasificación subjetiva en leve, moderado, abundante y un tipo especial denominado liquenoide. Este último consistió en una severa infiltración inflamatoria mixta que oscureció la unión dermoepidérmica. En este sentido, el infiltrado inflamatorio moderado fue el más observado $(28,2 \%)$.

El infiltrado inflamatorio de tipo liquenoide, que caracteriza a enfermedades como el lupus, se observó en el $18,8 \%$ de los casos. La incontinencia pigmentaria, otro hallazgo también citado con frecuencia, se encontró en el $35,3 \%$ de los cortes y el $40 \%$ correspondió a pacientes con LED (Tabla 3).

\section{DISCUSIÓN}

Las dermatosis autoinmunes en caninos son infrecuentes y de muy baja presentación clínica dentro del total de enfermedades que afectan a la piel ${ }^{6}$. En la bibliografía se estima que las dermatosis autoinmunes representan solo el $1,4 \%$ de las dermatosis caninas ${ }^{5}$, ${ }^{7}$. En el presente trabajo las dermatosis autoinmunes representaron el $2,07 \%$ del total de casos, siendo este valor cercano a los descriptos por diferentes autores.

Las afecciones cutáneas autoinmunes con mayor frecuencia diagnóstica en perros son el PF $(0,6 \%)$ y el $\operatorname{LED}(0,5 \%)^{1}$. Los resultados aquí obtenidos se corresponden con la bibliografía, ya que observamos un porcentaje similar para PF $(0,5 \%)$ y LED $(0,4 \%)$.

$\mathrm{Al}$ igual que lo observado por otros autores, no hemos encontrado diferencias significativas entre la presentación de la enfermedad en machos y hembras ${ }^{8,9}$. En el mismo sentido, constatamos que el cocker spaniel y el labrador retriever fueron dos de las razas que más frecuentemente fueron afectadas por $\mathrm{PF}^{7}$. También coincidimos en que la raza collie fue la que registró la mayor frecuencia de $\mathrm{LED}^{2}$. En relación a la edad, la bibliografía consultada establece el inicio de las lesiones antes de los 5 años, dato que se corresponde con nuestro análisis estadístico (media: 5,14 años) ${ }^{3,6}$.
Durante el examen clínico, la observación de pústulas y vesículas constituye un hallazgo característico de las enfermedades autoinmunes. Sin embargo, en nuestro estudio solo se observaron estas lesiones en un porcentaje menor al 5\%. Ello podría explicarse debido a que las mismas tienen un carácter transitorio producto de su fragilidad y rápida evolución a lesiones secundarias como úlceras y costras 9,10 .

En nuestro estudio pudo observarse que las costras $(43,5 \%)$ y úlceras $(31,8 \%)$ fueron las lesiones más registradas en el examen clínico. Cabe resaltar que en la clínica éstas son lesiones muy inespecíficas.

Los hallazgos microscópicos de acantosis, espongiosis y acantólisis con formación de pústulas que contienen queratinocitos acantolíticos en su interior, permiten identificar a las enfermedades del complejo pénfigo. Estos hallazgos se deben a la falta de adhesión entre los queratinocitos. A diferencia de lo que sucede con las pústulas macroscópicas, las pústulas microscópicas se observaron en más del $50 \%$ de los casos, variando su ubicación en los diferentes estratos de la epidermis, según el tipo de pénfigo.

La identificación y ubicación de las pústulas, así como su asociación con la acantólisis, son hallazgos de importancia relevante en el diagnóstico histopatológico de las enfermedades que componen el complejo pénfigo.

En los casos de LED los datos más frecuentes de carácter diagnóstico son la presencia de queratinocitos apoptóticos, infiltrado inflamatorio de tipo liquenoide, incontinencia pigmentaria, degeneración vacuolar de los estratos granuloso y basal, como así también áreas de separación dermoepidérmica. Estos hallazgos están relacionados con la formación de autoanticuerpos que, cuando se unen a antígenos, liberan inmunocomplejos que se depositan en la zona de membrana basal.

En nuestro estudio, entre los hallazgos más frecuentes, pudimos observar la separación dermoepidérmica $(45,9 \%)$, la incontinencia pigmentaria $(35,3 \%)$, el infiltrado de tipo liquenoide $(18,8 \%)$ y los queratinocitos apoptóticos $(18,8 \%)$, siendo estos resultados coincidentes con los descriptos por otros autores ${ }^{3,10}$. 
Se concluye que las dermatosis autoinmunes son de muy baja presentación dentro del total de enfermedades que afectan a la piel de caninos. No obstante, estas afecciones deben ser tenidas en cuenta e incluidas en los diagnósticos diferenciales de aquellos pacientes que se presentan a consulta clínica/dermatológica con lesiones cutáneas como pústulas, vesículas, bullas y -más frecuentemente- costras, erosiones y úlceras.

Asimismo, es importante resaltar la existencia de una mayor predisposición en animales de raza pura, siendo collie, pastor alemán, cocker spaniel y labrador retriever las más predispuestas. Las lesiones son más comúnmente observadas en la piel y mucosas de la cara y secundariamente en el resto del cuerpo.

La histopatología resulta un método útil para el diagnóstico de estas enfermedades y, en nuestro medio, sigue siendo la herramienta más utilizada por los clínicos y dermatólogos para arribar a un diagnostico definitivo en tales afecciones. No obstante, nuevas estrategias diagnósticas están siendo aplicadas en la actualidad como las técnicas de inmunohistoquímica y de inmunofluorescencia directa e indirecta, que permiten evidenciar los diferentes autoanticuerpos y antígenos presentes en cada enfermedad y su ubicación sobre la piel, permitiendo diferenciarlas unas de otras.

Agradecimientos. A la MV MSc Laura Alarcón por su colaboración en el análisis de datos estadísticos y a las histotecnólogas Carolina Aralda y Lorena Díaz (FCV-UNLP), por su ayuda en el procesamiento patológico.

\section{REFERENCIAS}

1. Bedolla MA, Salas GG, Constantino CF. 2009. Diagnóstico inmunohistoquímico de dermatosis inmunomediadas en perros domésticos. Vet Mex 40: 181-189.

2. Goo MJ et al. 2009. Discoid lupus erythematosus (DLE) in a spitz dog. J Vet Med Sci 70: 633-635.

3. Gross TL, Ihrke PJ, Walder EJ, Affolter VK. 2005. Inflammatory, dysplastic, and degenerative diseases. In: Skin diseases of the dog and cat (Gross TL et al. Edit.), $2^{\circ}$ ed., Blackweel Publishing, New Jersey, p. 555.

4. Hargis AM, Myers S. 2017. The integument. In: Pathologic basis of veterinary disease (Zachary JF, McGavin MD, Edit.), $6^{\circ}$ ed., Elsevier, St. Louis, Missouri, p. 1009-1146.

5. Larsson C. 2005. Wandering through the autoimmune dermatoses: pemhpigus complex. In: Proceedings of the World Small Animal Veterinary Ass., Mexico City,: http:// www.vin.com/apputil/content/defaultadv1.aspx?pId=1119 $6 \&$ meta $=$ Generic \&id $=3854248$

6. Mauldin EA, Peters KJ. 2016. Integumentary system. In: Pathology of domestic animals (Jubb KV, Kennedy PC, Palmer NC, Edit.), $6^{\circ}$ ed., Elsevier, St.Louis, Missouri, vol. 1, p. 509-736.

7. Miller WH, Griffin CE, Campbell KL. 2014. Dermatosis autoinmunes e inmunomediadas. En: Dermatología en pequeños animales (Muller \& Kirk Edit.), $3^{\circ}$ ed., Intermédica, Buenos Aires, p. 478-552.

8. Olivry T, Chan LS. 2001. Autoimmune blistering dermatoses in domestic animals. Clin Dermatol 19: 750-760.

9. Olivry T. 2006. A review of autoimmune skin diseases in domestic animals: I-superficial pemphigus. Vet Dermatol 17: 291-305.

10. Olivry T, Linder KE. 2009. Dermatoses affecting desmosomes in animals: a mechanistic review of acantholytic blistering skin diseases. Vet Dermatol 20: 313-326. 